\title{
Phytochemical profiles and biological activities of Curcuma species subjected to different drying methods and solvent systems: NMR-based metabolomics approach
}

\begin{abstract}
Curcuma species (Zingiberaceae) have been used in traditional medicine in India and Southeast Asia to treat many human ailments and are believed to possess many biological activities. In this study, four Curcuma species (C. zedoaria, C. xanthorrhiza, C. aeruginosa and $C$. mangga) were compared in terms of their total phenolic contents (TPC), and bioactivities including free radical scavenging, $\alpha$-glucosidase and nitric oxide (NO) inhibition. Their phytochemical constituents were also determined by a proton nuclear magnetic resonance $\left({ }^{1} \mathrm{H}\right.$ NMR) based metabolomics approach. The species were dried using three drying methods (air, freeze and oven) and extracted with two ethanol ratios (50 and 100\%). The correlations between TPC and bioactivities, with phytochemical constituents were obtained using partial least square (PLS) regression. The results showed that $C$. xanthorrhiza had the highest TPC, antioxidant and NO inhibitory activities, whereas $C$. mangga showed the highest $\alpha$-glucosidase inhibitory activity. Notable and clear separations among the four species and the three drying methods for $C$. xanthorrhiza and $C$. mangga were revealed by principal component analysis (PCA). The PCA scores plot showed close relationship between $C$. xanthorrhiza and $C$. mangga, and were separated from $C$. zedoaria and $C$. aeruginosa. The PLS model indicated that curcumin, xanthorrhizol, 1-(4-hydroxy-3,5dimethoxyphenyl)-7-(4-hydroxy-3-methoxyphenyl)-(lE,6E)-1,6-heptadiene-3,4-dione and demethoxycurcumin were significantly correlated with the higher antioxidant and NO inhibitory activities as demonstrated by $C$. xanthorrhiza. Meanwhile, zerumin $\mathrm{B}$, dehydrocurdione, (E)-labda-8(17),12-diene-15,16-dial, calcaratarins A and curcuzederone were present in $C$. mangga contributed to the $\alpha$-glucosidase inhibitory activity. The freeze dried samples of all the absolute ethanol extracts of Curcuma species exhibited significant bioactivities with the highest contents of metabolites.
\end{abstract}

Keyword: Curcuma species; Multivariate data analysis; Nuclear magnetic resonance; Antioxidant; $\alpha$-Glucosidase inhibition; Nitric oxide inhibition 